\title{
Method for detection and quantification of non-invasive skin sympathetic nerve activity
}

\author{
Chun Liu ${ }^{1,2}$, Johnson Wong ${ }^{1}$, Anisiia Doytchinova ${ }^{1,3}$, Peng-Sheng Chen ${ }^{1}$, Shien-Fong Lin ${ }^{1,4}$
}

\begin{abstract}
Sympathetic nerve activity is an important trigger of cardiac arrhythmia. Our laboratory recently developed a new method for non-invasive recording of the skin sympathetic nerve activity (SKNA) using conventional electrocardiography (ECG) patch electrodes. Recent studies showed that SKNA can adequately estimate sympathetic tone in humans. In order to improve the analysis of SKNA, we developed automatic standard assessment system based on the concept of microneurography and applied to non-invasive SKNA recording via 4 steps of signal processing. Every parameters in procedures can be manually selected by users in order to meet requirements. After using these signal processing, the results obtained by this system show improved nerve burst morphology and trend. Intuitive nerve discharge pattern and occurrence frequency can be automatically generated. Verification of the method with the cold water pressor test data further established the reliability and usefulness of the system. This new method offered a convenient tool to evaluate SKNA for comprehensive and detailed application in neurocardiology. The analysis system may facilitate the study between SKNA and cardiac arrhythmia, thus advance the field of neurocardiology research.
\end{abstract}

Keywords - Microneurography, Skin Sympathetic Nerve Activity, Nerve assessment system, Cold water pressor test

\section{INTRODUCTION}

The sympathetic nervous system (SNS) which is one of the main divisions of the autonomic nervous system, functions to regulate the body's unconscious actions like heart beating. However, studies of estimating sympathetic tone are either invasive or require proper sinus node function. One method was microneurography. A fine tungsten needle electrode was inserted into the peripheral nerves to directly record sympathetic nerve activity[1]. This method of recording is difficult to perform, and cannot be used to study ambulatory subjects. While it is possible to record stellate ganglion nerve activity in animal models [2], those methods are not feasible for human subject research. Therefore, we have recently developed a method to record the skin sympathetic nerve activity (SKNA), which was proven to be effective in estimating the sympathetic tone [3-7]. Subsequent studies

\footnotetext{
${ }^{1}$ All authors are affiliated with the Krannert Institute of Cardiology, Division of Cardiology, Department of Medicine, Indiana University School of Medicine, Indianapolis, Indiana, USA.

${ }^{2}$ Chun Liu is with Department of Electrical and Computer Engineering, National Chiao Tung University, Hsinchu, Taiwan (e-mail: jim0920335.eed04g@g2.nctu.edu.tw).

${ }^{3}$ Anisiia Doytchinova is with the Division of Cardiovascular Health and Disease, University of Cincinnati Medical Center, Cincinnati, OH

${ }^{4}$ Shien-Fong Lin, $\mathrm{PhD}$ is with Institute of Biomedical Engineering, College of Electrical and Computer Engineering, National Chiao Tung University, Hsinchu, Taiwan (corresponding author, phone: $+886-3-5131386$ e-mail: 1insf5402@nctu.edu.tw )
}

showed that the morphology and frequency of SKNA are important in triggering cardiac arrhythmia $[3,8,9]$. Current methods of analyzing SKNA [4] focused on examining the original filtered SKNA or the average SKNA over a period of time. A limitation of original filtered SKNA is that the frequency of nerve discharges cannot be determined, and that the onset and termination of a burst is difficult to determine. The average SKNA curve provides a rough shape of nerve activity. It does not help investigators determine the detailed nerve discharge patterns. Summarized the limitation above, it is hard for researchers to focus on the pattern and strength of nerve discharge. In order to solve these problem and broke through the limitation of current analysis, a novel assessment system of SKNA studies is needed. The purpose of the present study was to develop an automatic standard assessment system to quantify the changes and morphology of SKNA burst and relate the results to physiological events. Successful development of this system will offer researchers an intuitive nerve burst quantification, thus help investigators determine the trend, duration, amplitude and pattern of SKNA discharges. This information will then be used to advance cardiac arrhythmia research related to cardiac nerve function.

\section{METHOD}

\section{A. Signal Filtering}

The first step of signal processing is multiple filtering settings. To reject $\mathrm{A} / \mathrm{C}$ interference, the raw signal passes through a 50 to $70 \mathrm{~Hz}$ bandstop filter (8th-order Butterworth filter), if necessary. The recorded signal is filtered with a bandpass filter with a passband of 0.5 to $150 \mathrm{~Hz}$ to show the ECG. An ECG R-wave peak detector was implemented to detect the heart rate. Furthermore, the raw signal also passes through a pass-band with cutoffs at 500 and $1000 \mathrm{~Hz}$ (8-order Butterworth filter) to show filtered SKNA (fSKNA). The amplitude of fSKNA is around $\pm 60 \mu \mathrm{V}$.

From the R-R interval of each heartbeat, the beat per minute (BPM) index can be calculated and shown as a BPM curve.

$$
\begin{gathered}
R=\left[r_{0}, r_{1}, \ldots, r_{n}\right] \\
B P M=\frac{60 \times S R}{r_{i+1}-r_{i}}
\end{gathered}
$$

Where $\mathrm{R}$ is the array of detected R peak location, $\mathrm{SR}$ is Sampling Rate of data.

\section{B. Signal Rectification}

The goal of this assessment system is to identify the nerve discharge pattern of fSKNA. Full-wave rectification is applied 
after the initially filtered fSKNA so that all data points can be accumulated despite their polarity.

\section{Signal Smoothing-Moving Average (MA)}

Moving average (MA) of the rectified fSKNA is performed to generate a linear envelope of the signal [10]. The input data that pass through the procedure of rectification is represented as a one-dimension array $X$.

$$
X=\left[x_{0}, x_{1}, \ldots, x_{n}\right]
$$

In this process, we use $100 \mathrm{~ms}$ as window size and $50 \mathrm{~Hz}$ as the moving frequency, i.e., the window advances every $20 \mathrm{~ms}$. In the process, we calculate the average of all signal values in the selected window, and then, move the window to the next $20 \mathrm{~ms}$, repeats the steps until the end of the data so that MA is still a one-dimension array which represents the result of each window (Fig. 1a). The equation of moving average is:

$$
\begin{gathered}
M A=\left[m a_{0}, m a_{1}, \ldots, m a_{j}\right] \\
m a_{a}=\frac{\sum_{i=a}^{m+a-1} x_{i}}{m} \\
a=0,1, \ldots, j \\
j=\frac{(S R \times T-m) \times M_{\text {freq. }}}{S R}
\end{gathered}
$$

Where $j$ is the number of samples of MA, $m$ is the number of samples in a window; $x_{i}$ is the $i^{\text {th }}$ sample value of array $\mathrm{X}$; $S R$ is Sampling rate of data; $M_{\text {freq. }}$ is moving frequency of window; $T$ is the duration of selected segment in second.

The output of this procedure is defined as the moving average SKNA (mSKNA). After the MA operation, signal was effectively downsampled 200 times, and the number of data points was also reduced by 200 times.

\section{Signal Smoothing-Root Mean Square (RMS)}

Root mean square (RMS) process the signal to show the strength of signal that reflects the changes of amplitude in time domain.

Because nerve activity is a high-frequency signal, after the

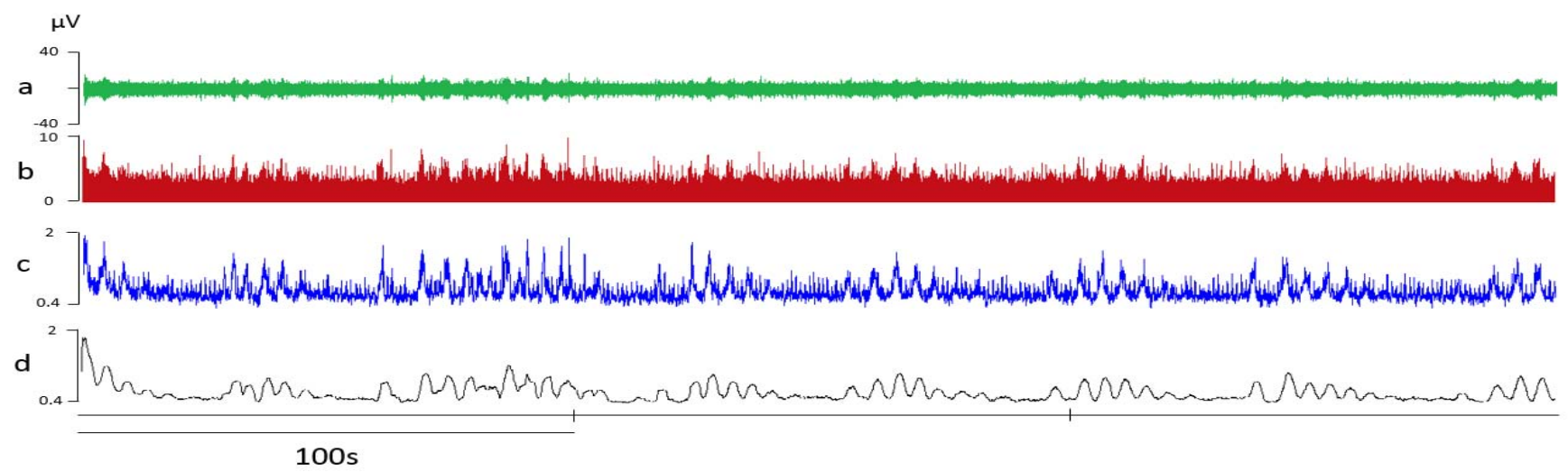

Figure 2. Signal processing. a: fSKNA signal, after 500-1000 Hz band-pass filter to present filtered SKNA signal; b: Rectified SKNA signal; c: mSKNA signal, a linear envelope was created by using moving average and the extreme parts of the signal was included; d: eSKNA after RMS operation. application of MA, the curve of signal is still jagged, making it difficult to confidently identify burst patterns. Therefore, we pass the mSKNA through an RMS calculator. In the current setting, we used 100 samples as window size and move the window every sample to avoid downsampling again (Fig.1b). The equation of RMS is similar to moving average. Equations (6), (7) still apply.

$$
\begin{gathered}
R M S=\left[r m s_{0}, r m s_{1}, \ldots, r m s_{j}\right] \\
r m s_{a}=\sqrt{\frac{\sum_{i=a}^{n+a-1} m a_{i}^{2}}{n}}
\end{gathered}
$$

Where $j$ is the number of samples of RMS, $r m s_{a}$ is the $a^{\text {th }}$ sample value of RMS; $n$ is the number of samples in a window; $m a_{i}$ is the $i^{\text {th }}$ element of moving average array.

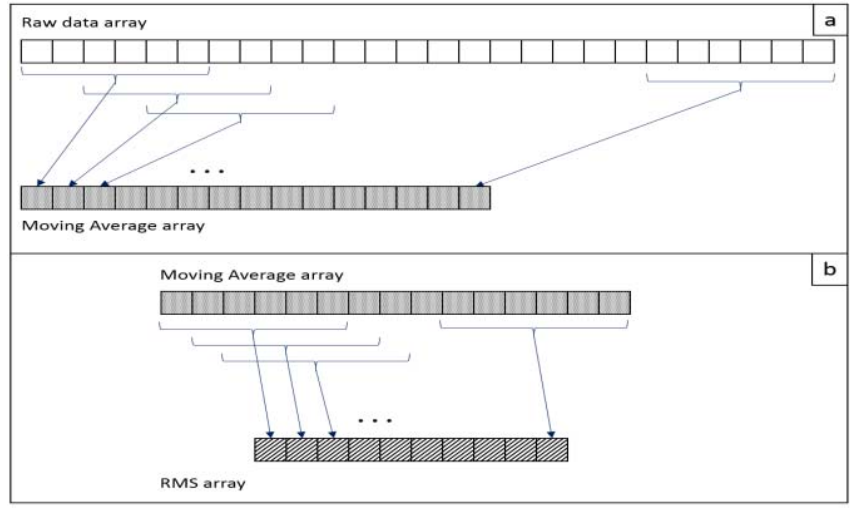

Figure 1. Schematic diagram. a: Moving Average procedure; b: RMS procedure

After the procedure, RMS signal is a one-dimension array which represents the main trend of nerve activity. We defined this envelope curve of SKNA as "eSKNA" which can more easily show periods of nerve activation in any given period (Fig. 2).

\section{E. Binary Time Series}

In order to provide intuitive graph interpretation, binary time series graph can help users efficiently distinguish the occurrence frequency of nerve burst in the selected period. We 
used equations (10) and (11) to determine the threshold.

$$
\begin{gathered}
\text { Baseline }=\text { average of lower } 20 \% \text { samples } \\
\text { Threshold }=(\text { Baseline }- \text { Min }) \times 5+\text { Min }
\end{gathered}
$$

Where Baseline is the average of lower $20 \%$ data points in selected window.

It can also be defined by users according to the appropriate range of eSKNA amplitudes. Once the threshold for nerve burst is set by the user, the eSKNA will be represented as a binary series, in which the durations where eSKNA are above the threshold are 1 (black) and under threshold 0 (white) (Fig. $3 b$ ). Without applying the RMS, this binary series may falsely indicate consecutive short on/off periods due to multiple crossing of the threshold.

\section{F. Intensity Graph}

To provide an alternative visualization method of eSKNA pattern, we developed the intensity graph to show the strength of nerve activity. The amplitude of eSKNA is converted into color gradient. The bright red part means strong activation of nerve signal and the dark black part presents the lack of nerve activity (Fig.3c).

The parameters of intensity graph also can be customized, in order to present an optimized color distribution.

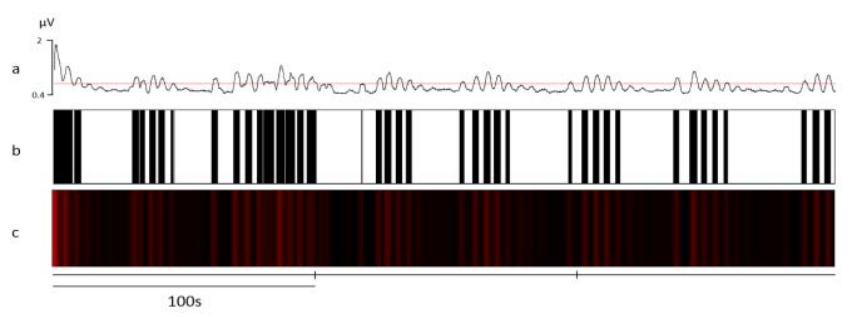

Figure 3. a: eSKNA of specific episode, with threshold in red; b: Binary Time Series graph; c: Intensity graph

\section{EXPERIMENTAL DESIGN}

We analyzed two sets of data obtained from human subjects. The human subject study protocols have been approved by the Institutional Review Board of Indiana University. The first part is healthy volunteers for SKNA recording during the cold water pressor test $(\mathrm{CPT})$. The data were from the same 12 patients included in a previous manuscript [3]. The purpose is to verify the output signal curve of the analysis to present a real activated nerve burst. Because CPT is a standard procedure to trigger sympathetic discharges [11], the CPT group is used as positive control for nerve discharges. [3, 8, 9]

The CPT subjects' data were recorded by using standard ECG patch electrodes (Fig. 4a) connected to a bioamplifier ML138 (ADInstruments, Colorado Springs, CO) recorder with a wide bandwidth $(5 \mathrm{kHz})$ and high sampling rate $(4 \mathrm{kHz} / 10 \mathrm{kHz})$. The acquired data were stored in the flash memory (CF memory card) of ML138 and transferred to the computer. Laboratory Virtual Instrument Engineering Workbench (LabVIEW) is a system-design platform and development environment for a visual programming language from National Instruments (Austin, TX). We used LabVIEW as the developing platform and designed a customer program for data analysis system called NerveAct.

The NerveAct provided clear and easy-to-use graphical user interface (GUI) (Fig. 5) and allowed users to input the raw digitized acquisition data and processed using customized parameters. There are three individual windows which present acquired data in different leads. The bottom window shows the R-peak detection and R-R interval analysis. The filtration and other signal processing can be set from the side of each windows. The duration parameter let users set the duration in one window and the arrows on the top provide fine adjustment of windows. After the data pass through the procedures of program, we can obtain an output representing eSKNA and other skin nerve-related information.

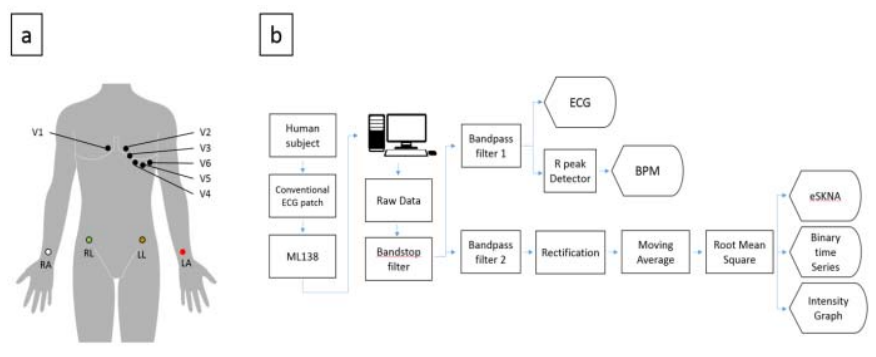

Figure 4. Setting of data acquisition. (a) ECG leads configurations for CPT (b) Flow chart of signal processing

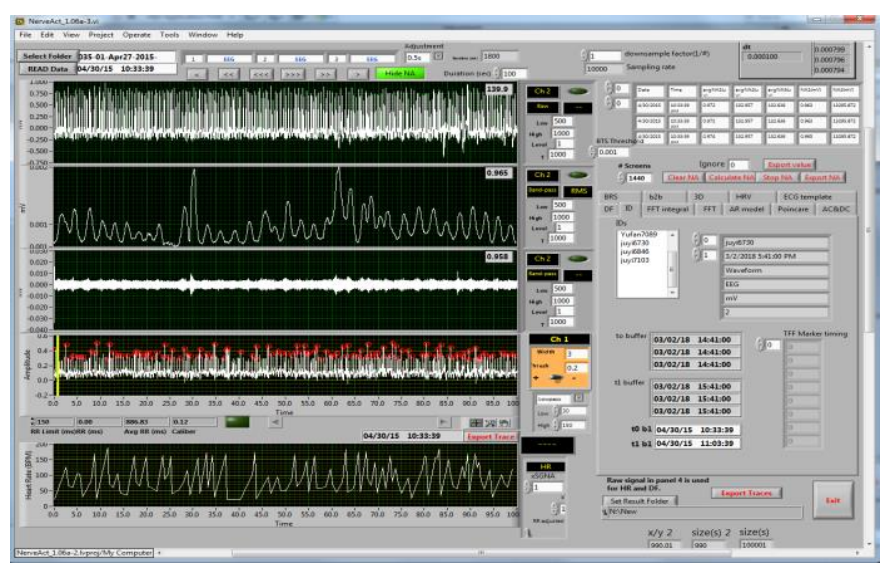

Figure 5. GUI of NerveAct

\section{RESULTS}

Using the custom-designed program, we can easily detect the difference between the original filtered SKNA and eSKNA. The eSKNA, which ignores the extreme part of nerve signal, presents an envelope of filtered SKNA signal. It can reveal a clear and marked nerve discharge compared to original filtered SKNA. The nerve discharge pattern has been neglected in the past due to the difficulties in its characterization. It can now be identified with the method developed in this research. The new method of assessment system can conveniently enhance the recognition of skin nerve discharge. Verification with the cold water pressor test data, the reliability and usefulness of the system are further supported . 
TABLE I shows the recording conditions and sampling rate in the experiment which include CPT $(\mathrm{N}=12)$.

TABLE I. RECORDING CONDITIONS AND SAMPLING RATE

\begin{tabular}{|l|l|l|l|}
\hline Patients & Sampling Rate & ECG Lead & Protocol \\
\hline $1-10$ & $4 \mathrm{kHz}$ & $\mathrm{I}, \mathrm{II}, \mathrm{V} 1-\mathrm{V} 6$ & Health volunteers in CPT \\
\hline $11-12$ & $10 \mathrm{kHz}$ & $\mathrm{I}, \mathrm{II}, \mathrm{V} 1-\mathrm{V} 6$ & Health volunteers in CPT \\
\hline
\end{tabular}

Fig. 6 shows the raw signal and the results after various processing from one of the healthy CPT volunteers. The SKNA from this subject was significantly activated in the CPT duration compared to the preparing and recovery period. Dotted green lines mark start and stop of CPT. This phenomenon provides a strong evidence to verify sensitivity of the system. So it has further verified that we can use the NerveAct as the tool to survey SKNA in cardiac arrhythmia patients.

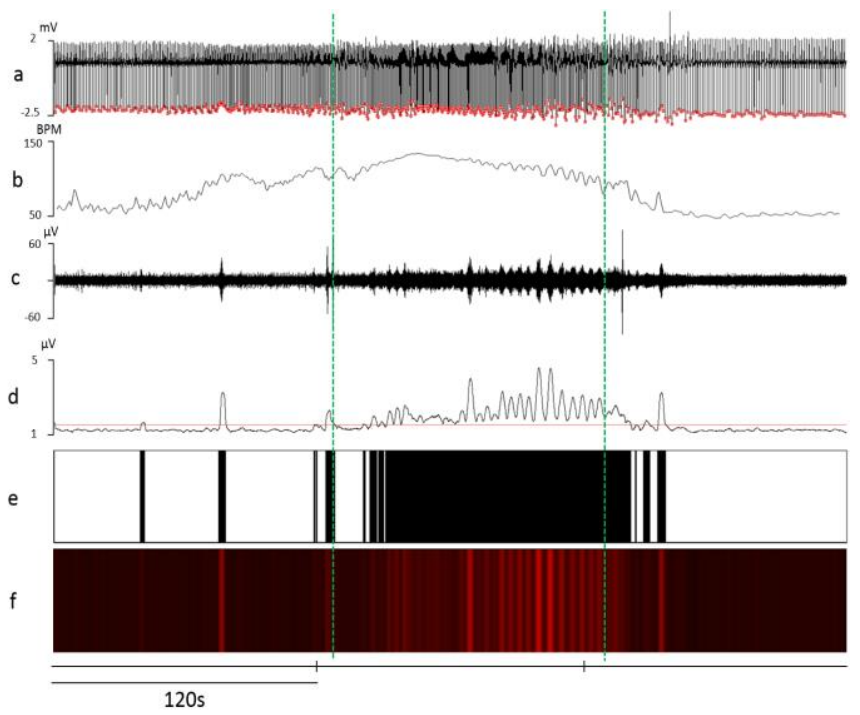

Figure 6. System outputs of CPT subjects. Dotted green lines mark start and stop of CPT. a: ECG and R peak detection; b: BPM; c: SKNA; d: eSKNA; e: Binary Time Series; f: Intensity Graph

Compared to the original SKNA, the nerve bursts locations in eSKNA curve are more distinct from baseline. The output curve eSKNA, binary time series, and intensity graph help users to more confidently identify nerve bursts for further analysis or diagnosis.

\section{CONCLUSION \& DISCUSSION}

Microneurography studies are standard methods to analyze sympathetic discharges. The signals are typically integrated to detect individual bursts. The amplitude and the frequencies of the nerve burst activity are then used to determine the effects of intervention $[12,13]$. Different from frequency domain analysis of heart rate variability (HRV) that provides an index of sympathetic tone, SKNA has been reported to show real-time nerve activity signal and used to analyze specific episode of cardiac events. SKNA allows us to observe real nerve discharge signal and pattern instead of mathematically derived sympathetic tone. Because SKNA is a new method of nerve activity recording, it is highly desirable to convert the

raw SKNA signals into burst activities similar to the integrated microneurography signals. In this manuscript we report a new assessment system of SKNA. This new method helps researchers convert the signals into burst activities similar to that observed in microneurography studies. The converted nerve burst patterns can be useful for the future studies on nerve signal analyses to determine the causal relationship between SKNA and cardiac arrhythmia. In addition, because of the morphological similarity between eSKNA and that of the integrated microneurogram, it is possible to compare these two nerve activity recordings quantitatively. These methods will allow SKNA investigators to compare their results with microneurography investigators, thus unifying the field of nerve recording studies.

\section{REFERENCES}

K. E. Hagbarth and A. B. Vallbo, "Pulse and respiratory grouping of sympathetic impulses in human muscle-nerves," Acta Physiol Scand, vol. 74, pp. 96-108, Sep-Oct 1968.

B. C. Jung, A. S. Dave, A. Y. Tan, G. Gholmieh, S. Zhou, D. C. Wang, et al., "Circadian variations of stellate ganglion nerve activity in ambulatory dogs," Heart Rhythm, vol. 3, pp. 78-85, Jan 2006.

A. Doytchinova, J. L. Hassel, Y. Yuan, H. Lin, D. Yin, D. Adams, et al., "Simultaneous noninvasive recording of skin sympathetic nerve activity and electrocardiogram," Heart Rhythm, vol. 14, pp. 25-33, Jan 2017.

T. H. t. Everett, A. Doytchinova, Y. M. Cha, and P. S. Chen, "Recording sympathetic nerve activity from the skin," Trends Cardiovasc Med, vol. 27, pp. 463-472, Oct 2017.

E. A. Robinson, K. S. Rhee, A. Doytchinova, M. Kumar, R. Shelton, Z. Jiang, et al., "Estimating sympathetic tone by recording subcutaneous nerve activity in ambulatory dogs," $J$ Cardiovasc Electrophysiol, vol. 26, pp. 70-8, Jan 2015.

M. J. Shen and D. P. Zipes, "Role of the autonomic nervous system in modulating cardiac arrhythmias," Circ Res, vol. 114, pp. 1004-21, Mar 142014.

S. Zhou, B. C. Jung, A. Y. Tan, V. Q. Trang, G. Gholmieh, S. W. Han, et al., "Spontaneous stellate ganglion nerve activity and ventricular arrhythmia in a canine model of sudden death," Heart Rhythm, vol. 5, pp. 131-9, Jan 2008.

A. Uradu, J. Wan, A. Doytchinova, K. C. Wright, A. Y. Lin, L. S. Chen, et al., "Skin Sympathetic Nerve Activity Precedes the Onset and Termination of Paroxysmal Atrial Tachycardia and Fibrillation," Heart Rhythm, vol. 14, pp. 964-971, 20172017. R. A. Kabir, A. Doytchinova, X. Liu, D. Adams, S. Straka, L. S. Chen, et al., "Crescendo skin sympathetic nerve activity and ventricular arrhythmia," J Am Coll Cardiol, vol. 70, pp. 3201-3202, 20172017.

V. Pandey and V. K. Giri, "High frequency noise removal from ECG using moving average filters," in 2016 International Conference on Emerging Trends in Electrical Electronics \& Sustainable Energy Systems (ICETEESES), 2016, pp. 191-195. J. Fagius, S. Karhuvaara, and G. Sundlof, "The cold pressor test: effects on sympathetic nerve activity in human muscle and skin nerve fascicles," Acta Physiol Scand, vol. 137, pp. 325-34, Nov 1989.

E. C. Hart, G. A. Head, J. R. Carter, B. G. Wallin, C. N. May, S. M. Hamza, et al., "Recording sympathetic nerve activity in conscious humans and other mammals: guidelines and the road to standardization," Am J Physiol Heart Circ Physiol, vol. 312, pp. H1031-H1051, May 12017.

D. W. White, J. K. Shoemaker, and P. B. Raven, "Methods and considerations for the analysis and standardization of assessing muscle sympathetic nerve activity in humans," Auton Neurosci, vol. 193, pp. 12-21, Dec 2015. 